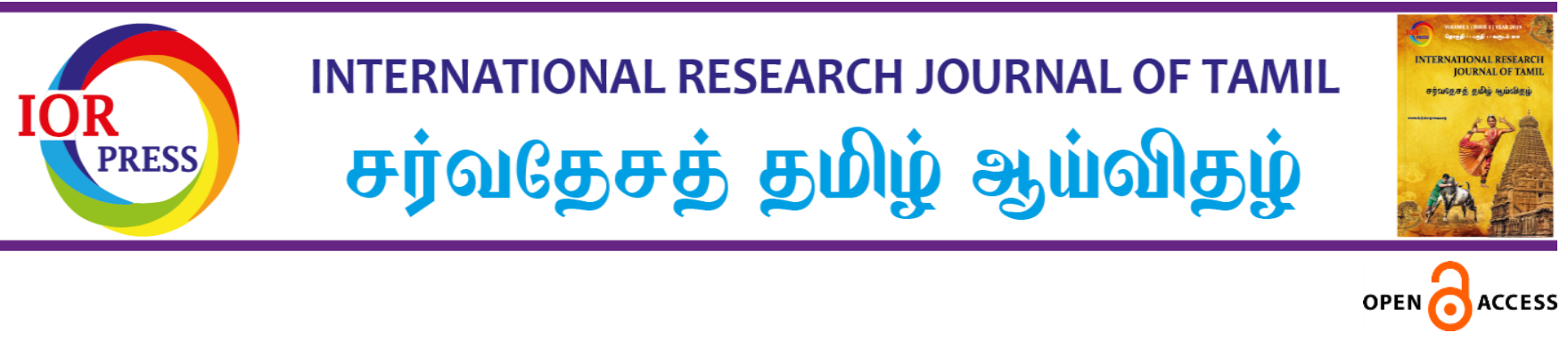

கதாநாயகி பெண் மன உணர்வியல்

செ. லிட்டில் ப்ளவர் அ, •

அ தமிழ்த்துறை, ஜெயராஜ் அன்னபாக்கியம் மகளிர் தன்னாட்சிக் கல்லூரி, பெரியகுளம், தேனி- 625601, தமிழ்நாடு, இந்தியா.

\title{
Female Psychology in Kathanayagi
}

\section{S. Little Flower a,}

a Department of Tamil, Jayaraj Annapackiam College for Women (Autonomous), Periyakulam, Theni- 625601, Tamil Nadu, India.

* Corresponding Author: littletamjac@annejac.ac.in

Received: 21-03-2021

Revised: 29-11-2021

Accepted: 07-12-2021

Published: 08-02-2022

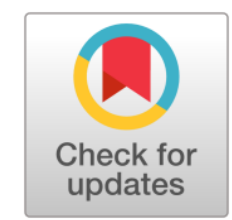

\begin{abstract}
In recent literary genres novels has got a unique identity. Novels describes about the autobiography, historical studies, real life incidents and these are the story line of novels. Novels are written as an attempt to solve social problems and the problems faced by women. Psychology can be defined as how an individual react in the Society and not only that but also his learning ability, memory power and the loss of memory. The basic thing which is responsible for the creation of literature is human thoughts and feeling. They create literature based on their female experiences or of what they learn from circumstances and emotions play a vital role in the novels. Since the characters exhibit a volume of emotions, there is a spontaneous flow of psychological impacts. Tamil literary books and Tamil novels are the outcome of mixed human feelings in various situations. This essay is an abstract of various feeling and thoughts of human like guilty, emotions and the hidden thoughts, written by famous novelist Aandal Priyadharshini.
\end{abstract}

Keywords: Novel, Feminism, Psychological Emotion, Feminist Introspection, Aandal Priyadharshini.

\section{முன்னுரை}

உள்ளத்தின் இயல்புகளையும், அவற்றின் செயற்பாடுகளையும் புகுந்து விளக்கும் அறிவியல் துறையினை 1960 - ஆம் ஆண்டு வரையில் உளவியல் என்னும் பெயரால் சுட்டினர். அதன்பிறகு முதன்மையும், தலைமையும் கருதி நடத்தைகளைப் பற்றிய அறிவியலே உளவியல் எனப்போற்றும் வழக்கம் உண்டாகியது. பெண்ணின் உணர்வுகள், அவற்றைச் சார்ந்த எண்ணங்கள், அதையடுத்த அவளின் செயல்பாடுகள், முடிவில் அதுவே பழக்க வழக்கமாக மாறி அவளது நடை, உடை, பாவனையாக அமைகிறது. இதுவே பெண்ணின் ஆளுமை வளர அல்லது அமைய வழிகோலுகிறது. புதைந்து கிடக்கும் பொருள் போல பெண்ணின் அடி மனதில் பல எண்ண அலைகள் உலா வருகின்றன. ஆனால் சில சமயம் மன வேதனை தாளாத போது அது கடுமையாகப் பொங்கி எழும் தன்மை வாய்ந்ததாக உள்ளது (Priyadarshini, 2003). ‘கதாநாயகி' என்ற புதினத்தில் வரும் பெண் கதாபாத்திரங்களின் மன உணர்வுகளை ஆராய்வதாக இக்கட்டுரை அமைந்துள்ளது. 


\section{உளவியல்}

மனிதன் மற்றொருவனின் எண்ணங்களைக் குறித்து ஆராயத் தொடங்கிய நிலையில் உளவியல் தோற்றம் பெற்றது எனலாம்.

ஆங்கிலத்தில் 'சைக்காலாஜி'(psychology) என்னும் சொல் 'உளவியல்' என்கின்ற தமிழ்ச் சொல்லிற்கு நேரானது. "சைக்கி (Psucho) லோகஸ் (Logus) ஆகிய இரண்டு சொற்களின் கூட்டாகவே சைக்காலஜி வழங்கப்படுகிறது. சைக்கி ஆன்மாவைக் குறிக்கிறது. லோகஸ் என்பது அறிவு என்ற பொருளைக் குறிக்கிறது. ஆன்மாவை ஆயும் அறிவியல் (Science of the Soul) என்பதை சைக்காலஜிக்குப் பொருள் என்பர்" (Santhanam,1978).

“உளவியல் ஓர் அறிவியல். அது மனிதனின் நடத்தையைக் கூர்ந்து நோக்கிப் பரிசோதனை போன்ற அறிவியல் முறைகளைக் கையாண்டு மனிதனின் நடத்தைக்கான அடிப்படை விதிகளை எடுத்துரைக்கின்றது"என சண்முகம் குறிப்பிடுவது ஆராய்வதற்குரியது (Bahavathi, 1989). இவற்றின் வாயிலாக மனிதனின் நடத்தைகளைப் பற்றியும் அவனது நடத்தைக்குள் மறைந்திருக்கும் எண்ணங்களையும் நாம் அறியலாம்.

மனித உள்ளம், உள்ளத்தைத் தன்னகத்தே கொண்டுள்ள உடல், உடலில் தோன்றும் உணர்வுகள், அந்த உணர்வுகளின் வெளிப்பாடாகத் திகழும் நடத்தை ஆகியவற்றைப் பற்றி ஆராய்வதே உளவியல் என்று வாட்சன் கூறுகின்றார்.

உள்ளத்தைப் பற்றிக் கூறுவதால் 'உளவியல்' என்றும் 'மனவியல்' என்றும், மனிதனின் நடத்தையைப் பற்றி ஆராய்வதால் நடத்தையியல் என்றும் பல பெயர்களில் இது சுட்டப்படுகிறது.

\section{பெண்ணிய உளப்பகுப்பாய்வு}

பெண்ணிய உளப்பகுப்பாய்வு என்பது பெண்ணின் மன உணர்வுகளைத் துல்லியமாகப் பகுத்து ஆயும் ஒரு திறனாய்வு முறையாகும்.

“பெண்களின் மன உணர்வுகளை, உள்ளக் குமுறல்களை, விடுபடவேண்டும் என்ற ஆவேசத்தைத் தன்னால் முடியும், சாதிக்க வேண்டும் என்ற நம்பிக்கையைத் தன்மீது பூசப்பட்ட பெண், தீண்டாமை தீட்டு, பலவீனம் மிக்கவள், இரண்டாம் தரமானவள் என்ற கற்பிதங்களை உடைக்க வேண்டும் என்ற உணர்வுகளை வெளிப்படுத்தும் பெண்ணின் பேச்சையும், எழுத்தையும், நடத்தையையும் பகுத்தாய்ந்து புரிந்து கொண்டு அவள் இவற்றை மீறிவர அவளை அவள் முழுமையாக அறிய உதவுவது பெண்ணிய உளப்பகுப்பாய்வாகும்" (Kancana, 2003).

ஆண், பெண் என்ற பால் வேறுபாடுகள், பாலின அடிப்படையிலான சமூகப் பாத்திரங்கள் ஆகியவற்றை நிர்ணயிக்கும் மொழி, சமூக மனப்பயிற்சி ஆகியவை பற்றிய விழிப்புணர்வுடன் இலக்கியப் பாத்திரங்களை ஆராய்வது பெண்ணிய உளப்பகு ப்பாய்வாகும்.

\section{கதாநாயகி}

‘கதாநாயகி’ என்ற குறும்புதினத்தின் ஆசிரியர் திருநெல்வேலி மாவட்டத்தில் உள்ள பாளையங்கோட்டையில் 1962 - ஆம் ஆண்டு பிறந்தார். கவிதை, சிறுகதை, புதினம், கட்டுரை, திறனாய்வு என இதுவரை பல படைப்புகளைப் படைத்துள்ளார். ‘எழுத்துலகச்சிற்பி', ‘கவிச்செம்மல்' என பல விருதுகளையும் பெற்றுள்ளார் (Priyadarshini, 2003).

‘கதாநாயகி - மகள், மனைவி, தாய் இதோடு 'Professional' என்கிற நான்குமுக பிரம்மாக்களாய் உலா வருகின்ற நீலாயதாட்சி, மைதிலி, பூரணி, பேச்சியம்மா ஆகியோரைப் பற்றியே உலா வருகின்றது. 


\section{பெண் மன உணர்வுகள்}

படைப்பாளி தான் அனுபவித்த நிகழ்ச்சிகளை மையமாக வைத்து இலக்கியம் படைப்பதால் உணர்வுகள் முக்கியத்துவம் பெறுகின்றன. உணர்வின் வெளிப்பாடாகக் கதாப்பாத்திரம் இருப்பதால் இயல்பாகவே அதில் உளவியல் இடம் பெறுன்கிறது.

நடத்தையைப் பாதிக்கக் கூடிய உடல் மற்றும் உளவியல் மாற்றங்களை ஏற்படுத்தும் ஒ ஒரு நிலையே உணர்ச்சி எனப்படும்.

\section{கோபம்}

மனிதர்கள் தங்களை சார்ந்தவர்களாலோ, சாராதவர்களாலோ நேரடியாக அல்லது மறைமுகமாக அவமதிப்பிற்குள்ளாகும் போதும் தாக்கப்படும் போதும் கோபமடைகின்றனர். பொதுவாக உளவியலாளர்கள் கோபத்தை மூன்று வகைகளாகக் கண்டறிந்துள்ளனர். அவை பதற்றத்துடன் கூடிய திடீர்கோபம், அமைதியான உள்நோக்கத்துடன் கூடிய கோபம், எப்போதும் எரிச்சல் அல்லது கடுகடுப்பாக இருப்பது என்பவையாகும்.

‘கதாநாயகி’ குறும் புதினத்தில் வரும் நீலாயதாட்சியின் அத்தை பேச்சியம்மாள், தன் அண்ணன் சண்முகம்பிள்ளையின் வீட்டைத் தனக்கு உரிமையாக்கிக் கொள்ள வேண்டும் என்ற எண்ணத்தில் தன்னுடைய இரண்டாவது மகனுக்கு நீலாயதாட்சியை மனைவியாக்க முயல அதை நீலாயதாட்சி மறுக்க “உன் கல்யாணத்துக்கு இந்த வீட்டைச் சீரா எழுதி வாங்கிட்டு, மூணு முடிச்சை உன் கழுத்தில் ஏத்திட்டுத் தான் இனிமே நகரப் போறேன்" என்று அரக்கியைப் போன்ற முகபாவத்துடன், பித்துப் பிடித்துக் கத்தினாள் பேச்சியம்மாள். தன்னுடைய அண்ணன் மகள் என்ற பாசம் இல்லாமல் சொத்திற்காக அவளை பழிவாங்கத் துடிக்கும் எண்ணத்தைப் பார்க்கும் போது பெண்ணே பெண்ணிற்கு எதிரியாக மாறுவதை பார்க்க முடிகின்றது (Priyadarshini, 2003).

\section{அழுகை}

கண்களிலிருந்து நீரை சிந்தும் உணர்ச்சி வசப்பட்ட நிலைதான் அழுகை. பொதுவாக அழுகை, இழிவு, இழவு, வறுமை, அசைவு ஆகியவற்றின் காரணமாகப் பிறக்கின்றன என்று தொல்காப்பியர் என்று கூறுகின்றர்.

நீலாயதாட்சியின் அக்கா மைதிலி மாமியார் கொடுமை, வரதட்சணைக் கொடுமைப் பற்றி கதறி அழுகின்றாள். "ஆட்டோ வாங்கித் தரச் சொல்லு. காசுமாலை வாங்கிப் போடச் சொல்லுன்னு புகுந்த வட்டிலே விரட்டல், வாய் செத்த புருஷன் - பேராசை புடிச்ச மாமியார், வறுமையான அப்பா நா எங்கேன்னு போய் முட்டிக்க. ஐயோ கடவுளே" என்று தன்னுடைய புகுந்த வீட்டின் கொடுமைகமை எண்ணியும், தன்னுடைய வாழ்க்கை நிலையையும் எண்ணி அழுகின்றாள் (Priyadarshini, 2003).

\section{ஏக்கம்}

ஏக்கம் நினைவுகளின் மூலம் தன்னை வெளிப்படுத்துவது ஆகும். இவ்வுணர்ச்சி மன உறவுகளால் தூண்டப்படுகின்றது. மனிதன் விரும்பும் ஏதோ ஒன்று அவனுக்குக் கிடைக்கக் கூடியது என்று தெரிந்திருந்தும், அது கைவரப் பெறாத போது ஏற்படும் உணர்வு தான் ஏக்கம்.

நீலாயதாட்சி ஆதிபகவனின் உறவை வெறும் நட்பு என்று நினைக்காமல் அதையும் தாண்டிய உறவு நிலை என்று நினைக்கின்றாள். ஆனால் ஆதிபகவனின் எண்ணம் என்ன? என்று அவளால் தீர்மானிக்க முடியவில்லை. "அவரின் முகத்திலிருந்து எதையும் புரிந்து கொள்ள முடியாமல் தவிப்பின் உச்சத்தில் உணர்ந்தாள் நீலாயதாட்சி. இந்த நட்பு வாழ்நாள் முழுசும் தொடராதா? இந்தப் புரிதல் வாழ்நாள் முழுசும் தொடராதா? இந்த அனுசரணை வாழ்நாள் முழுசும் தொடராதா?" என்று 


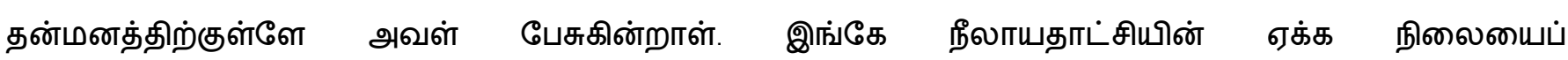
பார்க்கமுடிகின்றது (Priyadarshini, 2003).

\section{ஆதங்கம்}

மொழி ஞாயிறு தேவ நேயப் பாவாணர் அவர்கள் ஆதங்கத்திற்கு மனக்கவலை என்று பொருள் கூறுகின்றார். மைதிலி தன் புகுந்த வீட்டாரால் அவமதிப்பிற்குள்ளாகும் போதும், தன்னுடைய சுயத்தை இழக்கும் போதும், ஆதங்கப்படுகின்றாள். "ஒத்தைப் பைசா சுயமாகச் சம்பாதிக்கத் துப்பில்லே. தைரியமாய் வாழத் துப்பில்லே. எதுக்குமே பிரயோஜனமில்லாம எதுக்குப்பா என்னை வளர்த்தே? நாயும் நானும் ஒண்ணாச்சேப்பா. அடுத்த வேளைக்குப் புருஷன் சோறு போடுவானா? பட்டினி போடுவானான்னு தாலிகட்டினவன் கையை ஏக்கமாய்ப் பார்க்கற கொடுமை எனக்கு ேேணுமாப்பா?" (என்று மைதிலி தன் தந்தையைப் பார்த்து கேள்விக் கேட்கின்றாள் (Priyadarshini, 2003).

\section{மனச்சோர்வு}

"மனச்சோர்வு என்பது துன்பங்கள் ஊடுருவிய, தொடர்ந்து நீடித்துள்ள உணர்ச்சி வசப்பட்ட நிலையைக் குறிப்பன. அதிகச் சார்புதன்மை, நம்பிக்கை இழந்த மனநிலை தோல்வி மனப்பான்மை, மனக்கசப்பு, துயரங்கள், துக்கங்கள், உடல்நலக் கோளாறுகள் ஆகியனவற்றில் பாதிக்கப்பட்ட மனநிலையைச் சுட்டுவது, ஊக்கம், செயல்முறைகள், உணர்வுகள் ஆகியவை அதிகம் பிரதிபலிக்காமைக்கானக் காரணம் மனச்சோர்வே. உச்சக்கட்டம் கொடிய நிலையான மன அழுத்தம் எனப் பன்னாட்டு மகளிர் கலைக்களஞ்சியம் எடுத்துரைக்கிறது" (Nirmala Rani, 2003).

நீலாயதாட்சி தனக்கும் குமாருக்கும் இடையே நடைபெறவிருக்கின்ற கட்டாயத் திருமணத்தை நினைக்கும் போது அவளையும் அறியாமல் அவள் மனமானது பிறழ ஆரம்பிக்கின்றது. “திருமணம்கற பெயரிலே இந்த மாதிரி கொடுமை ஏதாவது நடந்தால் நிச்சயமாக ஹிஸ்டரிகலா ஆயிடுவேன். பைத்தியமா மாறிடுவேன். நட்பு இல்லாத வெறும் கல்யாணம் வேண்டாம். நேசம் இல்லாத வெறும் கல்யாணம் வேண்டாம். உடல் பசிக்கு மட்டுமே இரை போடற வெற்று தாம்பத்தியம் எனக்கு வேண்டாம். பொங்கவும், திங்கவும், தூங்கவும் மட்டுமே வாழ்க்கைனு சொல்ற ஆளோட இல்லறம் எனக்கு வேண்டாம், வேண்டாம், வேண்டவே வேண்டாம்" என்று அவள் குமுறுகின்றாள் (Priyadarshini, 2003).

\section{மகிழ்ச்சி}

மனநிறைவு ஏற்படும்போதும், வாழ்க்கை திருப்தி அடையும் போதும், மகிழ்ச்சி ஏற்படுகின்றது. மகிழ்ச்சி என்பது நீண்ட நாள் நல்வாழ்க்கைக்கான உணர்வு, அமைதி மற்றும் நமது வாழ்வோடு சேர்ந்த திருப்தி அதைத்தான் நாம் எல்லோரும் எல்லா நேரமும் தேடிக்கொண்டிருக்கிறோம்.

நீலாயதாட்சி, ஆதிபகவன் இருவரும் வாழ்வில் இணைகின்றர். தான் வாழ்க்கையில் எத்தனையோ இடர்பாடுகளை எதிர்க்கொண்டாலும் முடிவில் அவற்றையெல்லாமல் உதரி தள்ளி விட்டு தான் விரும்பிய வாழ்க்கையை அவள் தேர்ந்தெடுக்கின்றாள்.

“என் ஆதி பக்கத்திலே இருக்கும்போது என் சுவாசம் அர்த்தமுள்ளதாய்த் தான் இருக்கும். என் சந்தோஷத்தின் ஆதி அந்தமே நீதான் ஆதி. இந்த மாதிரியான அறிவுத் துணையைத் தான் திருமணத்தில் தேடினேன். பொக்கிஷமே கையில் கிடைச்சாச்சு என்று அவள் தன்னுடைய வாழ்க்கையை நினைத்து மகிழ்ச்சியடைகின்றாள் (Priyadarshini, 2003). 


\section{முடிவுரை}

பெண்ணியம் என்பது பெண்களின் வாழ்நிலை முன்னேற்றச் செயல்பாடுகள், அதில் ஏற்படும் தடைகள் மற்றும் தடைகளைக் களையும் முனைப்புகள் ஆகிய அனைத்தையும் உள்ளடக்கியதாகும். தற்காலச் சூழலில் புதினப் படைப்பில் பெண் படைப்பாளிகளின் பங்கு குறிப்பிடத் தக்க இடத்தினைப் பெற்றுள்ளது. பெண்களைச் சுற்றியுள்ள சிக்கல்கள், பெண்ணின் மீதான நிலைப்பாடுகள், இச்சமூகத்தில் பெண் என்பவள் எப்படிப்பட்டவளாகக் கருதப்படுகின்றாள், தன்னுடைய சுயத்தை எவ்வாறு தற்காத்துக் கொள்கின்றாள் என்பதை 'கதாநாயகி’ வழி ஆசிரியர் ஆண்டாள் பிரியதர்ஷினி தெளிவாகப் படம் பிடித்துக் காட்டியுள்ளார். உளவியலின் அடிப்படை ஆய்வு என்பது உள்ளுணர்வு அறியும் ஆற்றல், கவனம், மனஉணர்வு, உணர்ச்சி வேகம், இயல்பு ஊக்கம், மூளை செயல்பாடுகள், ஆளுமை நடத்தை மற்றும் உள்ளார்ந்த தொடர்புகள் ஆகியவற்றைக் கொண்டதாகும். பெண்ணானவள் முழு வளர்ச்சிப் பெற்றவளாக வளர வேண்டும் என்றால் அவளது உள்ளம் எத்தகைய சிக்கல்களுக்கும் ஆட்படாமல் இருந்தால் மட்டுமே முடியும். ஆனால் அப்பெண் தன் குடும்பத்தாரால் சமுதாயத்தினரால் பல நிலைகளில் சிக்கலுக்கு உள்ளாகின்றாள் என்பதை அப்பெண்ணின் கூற்று வழியாக இக்கட்டுரை எடுத்தியம்பியுள்ளது.

\section{References}

Bahavathi, K., (1989) Performance Approaches, World Tamil Research Institute, Chennai, India.

Kancana, R., (2003) Feminist Psychoanalysis and female language, Vishnupriya Publishing, Madurai, India.

Nirmala Rani V., (2003) Feminist Performance Review, Kavya Publishing, Chennai, India.

Priyadarshini, (2003) Kathanayagi, Kangai Book Station, Chennai, India.

Santhanam, S., (1978) The Psychological Basics of Education, Palaniappa Brothers, Chennai, India.

Funding: No funding was received for conducting this study.

Conflict of Interest: The Author has no conflicts of interest to declare that they are relevant to the content of this article

About the License:

\section{(ㄷ)(1)}

Attribution 4.0 International (CC BY 4.0)
(C) The Author 2022. The text of this article is licensed under a Creative Commons Attribution 4.0 International License 